\title{
AN APPROXIMATE EXPERIMENTAL METHOD FOR THE ANALYSIS OF EMF WAVES.
}

\author{
By P. G. Agnew. \\ 1. INTRODUCTION.
}

It is often of importance, both in physical and in engineering work, to obtain a knowledge of the harmonics present in the electromotive force wave of a generator. While several types of apparatus are commercially obtainable, notably oscillographs and curve tracers, which trace the wave with various degrees of accuracy, most of such apparatus is elaborate and expensive. After the curves are obtained the ordinates at a specified number of points are accurately measured and from the results the amplitudes of the various harmonics are calculated. The arithmetical computation of these Fourier coefficients has been greatly simplified within the last few years by methods developed by a number of different investigators. In some forms of apparatus the curve is not obtained automatically, but the ordinates are given directly by point to point methods. Of some 20 methods summarized by Orlich ${ }^{1}$, all but one, the method of resonance due to Pupin, ${ }^{2}$ require highly specialized apparatus.

Pupin passed the current to be analyzed through a noninductive resistance, around the terminals of which he connected his tuning circuit, consisting of a condenser and an adjustable inductance. By adjusting for resonance with any desired harmonic, and by observing the rise of potential across the condenser by means of an electrostatic voltmeter, it is possible to calculate the amplitude of the harmonic. But even in this case it is necessary to have at hand a relatively wide range of adjustable self-inductances and electrostatic voltmeters with a considerable range of sensitiveness.

${ }^{1}$ Orlich, Aufnahme und Analyse von Wechselstromkurven, Braunschweig, 1906.

${ }^{2}$ Am. Journal of Science, 48, pp. 379, 473; I 894. 
The experiments here described were undertaken to determine whether it is possible to get an approximate value of the first and second harmonics present in an emf. wave by means of ordinary portable instruments and calibrated condensers.

A distorted electromotive force wave will pass more current into a condenser than a pure sine wave having the same effective

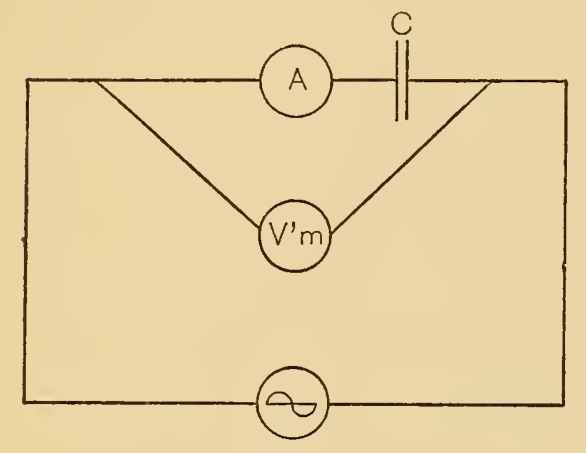

Fig. 1. value, since the effective rate of change of potential across the terminals of the condenser is greater for the distorted wave. We ought, then, to be able to use this fact to determine the amplitudes of the harmonics to which the distortion is due. For simplicity, suppose the third harmonic only to be present, and let $E$ be the reading of the voltmeter and $I$ that of the ammeter (Fig. I). If $E_{1}$ and $E_{3}$ represent the emfs of the fundamental and harmonic, then we may consider that they cause the respective currents $i_{1}$ and $i_{3}$ to flow in the condenser. If $C$ is the capacity and $p$ is 2 II times the frequency of the fundamental, and if we neglect the resistance of the ammeter,

$$
\begin{array}{ll}
i_{1}=E_{1} p C ; & E^{2}=E_{1}{ }^{2}+E_{3}{ }^{2} \\
i_{3}=E_{3} \cdot 3 p C ; & I^{2}=i_{1}{ }^{2}+i_{3}{ }^{2}
\end{array}
$$

we thus have four equations connecting $E_{1}, E_{3}, i_{1}, i_{3}$, from which these four quantities may be calculated. This is the inverse of the process sometimes employed in correcting for wave form, for example, in the 3 -voltmeter method of measuring inductance. Theoretically, it could be extended to any number of harmonics. Preliminary experiments on waves, obtained by combining emfs. of different frequencies in known proportions, indicated that the method was limited, by the unavoidable errors, to the determination of one harmonic, and that it did not give good results even then. Of course, account has to be taken of the resistance and inductance of the ammeter. It is evident that it is extremely 
difficult to obtain indicating instruments whose error is, for example, not greater than o.I per cent, under the conditions of frequency and wave form implied.

\section{THE METHOD USED.}

The necessity of knowing the absolute values of the current and voltage may be avoided by substituting a resistance in place of the condenser and adjusting its value to give the same settings on ammeter and voltmeter as were observed with the condenser. This is really making the ultimate measurement that of an impedance rather than that of an emf., as will be seen by developing the formula for the ratio of the harmonic to the fundamental emf. Suppose the third and the fifth harmonics present. Let $L$ (Fig. 2)

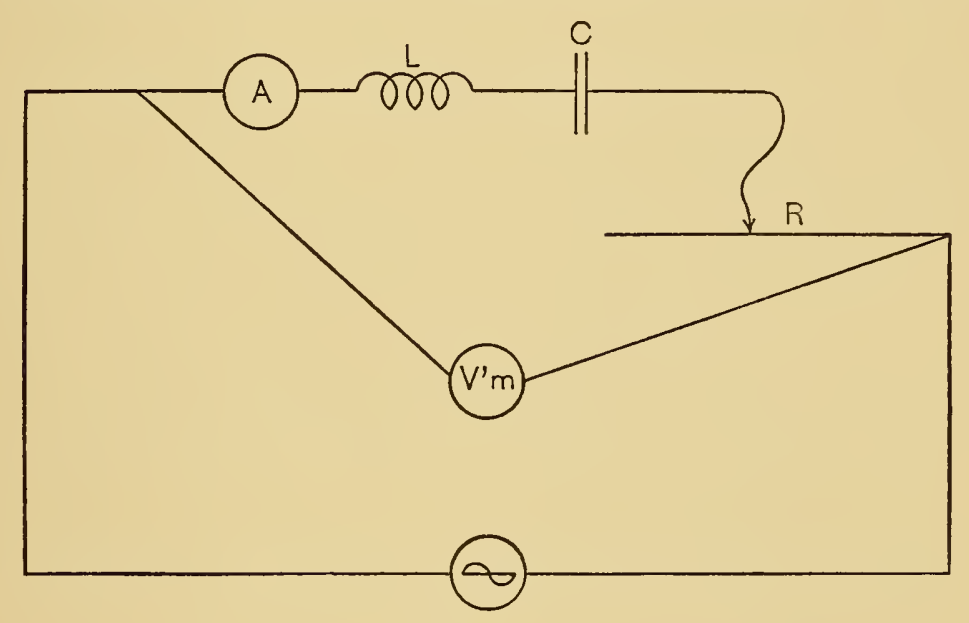

Fig. 2.

be the total inductance, and $R$ the total resistance of the circuit. Then the three components of current are

$\frac{E_{1}}{\sqrt{R^{2}+\left(p L-\frac{\mathrm{I}}{p C}\right)^{2}}}, \frac{E_{3}}{\sqrt{R^{2}+\left(3 p L-\frac{\mathrm{I}}{3 p C}\right)^{2}}}, \frac{E_{5}}{\sqrt{R^{2}+\left(5 p L-\frac{\mathrm{I}}{5 p C}\right)^{2}}}$

So that

$$
I^{2}=\frac{E_{1}{ }^{2}}{R_{1}{ }^{2}+\left(p L-\frac{\mathrm{I}}{p C_{1}}\right)^{2}}+\frac{E_{3}{ }^{2}}{R_{1}{ }^{2}+\left(3 p L-\frac{\mathrm{I}}{3 p C_{1}}\right)^{2}}+\frac{E_{5}{ }^{2}}{R_{1}{ }^{2}+\left(5 p L-\frac{\mathrm{I}}{5 p C_{1}}\right)^{2}}(\mathrm{I})
$$


where subscripts have been added to $R$ and $C$ to indicate that they are corresponding values. Now choose another value of $C$ and adjust $R$ so as to obtain the same settings of voltmeter and ammeter, giving

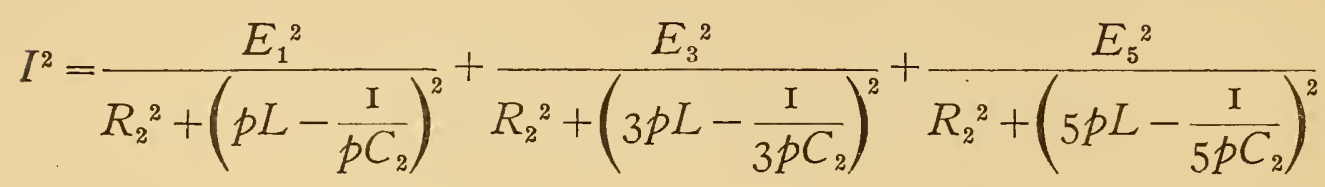

Finally, short circuit the condenser and again adjust $R$ to obtain the same settings, giving

$$
I^{2}=\frac{E_{1}{ }^{2}}{R_{3}{ }^{2}+p^{2} L^{2}}+\frac{E_{3}{ }^{2}}{R_{3}{ }^{2}+9 p L^{2}}+\frac{E_{5}{ }^{2}}{R_{3}{ }^{2}+25 p^{2} L^{2}}
$$

Equations (I), (2), and (3) suffice for the calculation of the ratios $E_{3} / E_{1}$ and $E_{5} / E_{1}$. Writing for brevity $a_{1}, a_{2}, \ldots \ldots a_{9}$ for the squares of the impedances in the nine denominators in the order given above, and comparing (3) successively with (I) and with (2),

$$
\left.\begin{array}{l}
\frac{E_{1}^{2}}{a_{1}}+\frac{E_{3}{ }^{2}}{a_{2}}+\frac{E_{5}^{2}}{a_{3}}=\frac{E_{1}^{2}}{a_{7}}+\frac{E_{3}{ }^{2}}{a_{8}}+\frac{E_{5}{ }^{2}}{a_{9}} \\
\frac{E_{1}^{2}}{a_{4}}+\frac{E_{3}{ }^{2}}{a_{5}}+\frac{E_{5}{ }^{2}}{a_{6}}=\frac{E_{1}^{2}}{a_{7}}+\frac{E_{3}{ }^{2}}{a_{8}}+\frac{E_{5}{ }^{2}}{a_{9}}
\end{array}\right\}
$$

Collecting terms, these become

$$
\begin{aligned}
& \left(\frac{\mathrm{I}}{a_{2}}-\frac{\mathrm{I}}{a_{8}}\right) E_{3}{ }^{2}+\left(\frac{\mathrm{I}}{a_{3}}-\frac{\mathrm{I}}{a_{9}}\right) E_{5}{ }^{2}+\left(\frac{\mathrm{I}}{a_{1}}-\frac{\mathrm{I}}{a_{7}}\right) E_{1}{ }^{2}=0 \\
& \left(\frac{\mathrm{I}}{a_{5}}-\frac{\mathrm{I}}{a_{8}}\right) E_{3}{ }^{2}+\left(\frac{\mathrm{I}}{a_{6}}-\frac{\mathrm{I}}{a_{9}}\right) E_{5}{ }^{2}+\left(\frac{\mathrm{I}}{a_{4}}-\frac{\mathrm{I}}{a_{7}}\right) E_{1}{ }^{2}=\mathrm{O}
\end{aligned}
$$

Replacing the six coefficients in the parentheses by $m_{1}, m_{2}, m_{3}, m_{4}$, $m_{5}, m_{6}$, respectively, we get

$$
\left.\begin{array}{l}
m_{1} E_{3}{ }^{2}+m_{2} E_{5}{ }^{2}+m_{3} E_{1}{ }^{2}=0 \\
m_{4} E_{3}{ }^{2}+m_{5} E_{5}{ }^{2}+m_{6} E_{1}{ }^{2}=0
\end{array}\right\}
$$


Solving for the ratios of the harmonics to the fundamental,

$$
\begin{aligned}
& \frac{E_{3}}{E_{1}}=\sqrt{\frac{m_{3} m_{5}-m_{2} m_{6}}{m_{2} m_{4}-m_{1} m_{5}}} \\
& \frac{E_{5}}{E_{1}}=\sqrt{\frac{m_{1} m_{6}-m_{3} m_{4}}{m_{2} m_{4}-m_{1} m_{5}}}
\end{aligned}
$$

In case it is desired to make the assumption that only one harmonic is present, we have only to put $E_{3}$ or $E_{5}$ equal to zero in the first equation of (4) giving, for the presence of the third harmonic only,

$$
\frac{E_{3}}{E_{1}}=\sqrt{\frac{-m_{3}}{m_{1}}}
$$

and for the presence of the fifth alone,

$$
\frac{E_{5}}{E_{1}}=\sqrt{\frac{-m_{3}}{m_{2}}}
$$

Theoretically the method might be extended to any number of harmonics, but the limitations of the accuracy of the readings and the greatly increased labor of computation limit it practically to two.

Should it be desired to solve for other harmonics instead of for the third and fifth, as in certain cases of polyphase work, the changes necessary in equations (I), (2), and (3) are obvious.

A vector diagram brings out the relations more clearly. The terms on the right of equation (I) are the squares of the component currents.

$$
I^{2}=i_{1}{ }^{2}+i_{3}{ }^{2}+i_{5}{ }^{2}
$$

Let the fundamental be represented by the $x$ coordinate of a system of space vectors (Fig. 3 ), $i_{3}$ by the $y$ and $i_{5}$ by the $z$ coordinate. The resultant vector, $I$, then represents the combined current. The relative magnitudes of the component currents are next changed by altering the constants of the circuit. The vector $I$ remains of the same length, but rotates to a new position. The constants of the circuit being again changed, the end of the vector $I$ again changes to a third position on a sphere of radius $I$. Hence the above algebraical solution is mathematically analogous to the 
solution of the spherical triangle determined by the three positions of the end of the vector $I$.

This graphical representation shows that the analysis of a much distorted wave will give values which are not only relatively, but absolutely, more accurate than that of a wave which is nearly sinusoidal. We are determining the sines of small angles by the changes in the cosines of the angle, and so the method can not be expected to yield reliable values of harmonics which are present

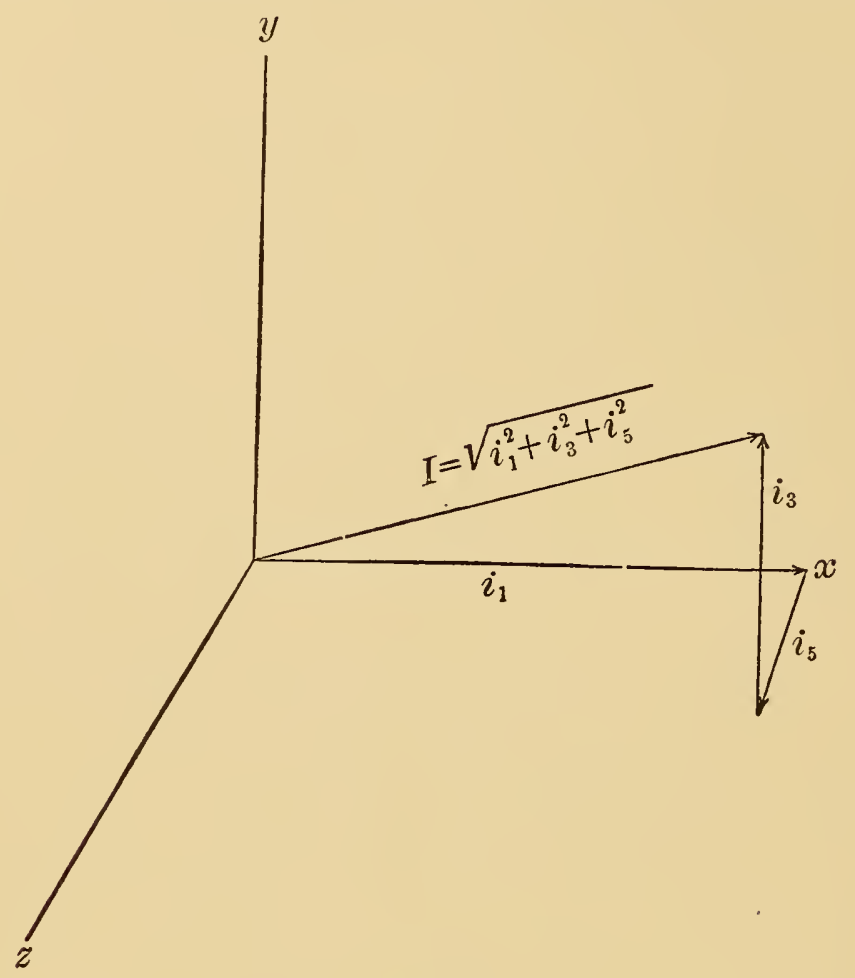

Fig. 3.

to the extent of only a per cent or so. Accordingly, if in a numerical calculation a component comes out as a small imaginary of the above order of magnitude, it should be taken to be zero so far as the accuracy of the method indicates.

\section{EXPERIMENTAL CONDITIONS FOR GREATEST ACCURACY.}

The quantities which enter into the calculation are resistance, inductance, and capacity, which depend directly on bridge measurements, together with the frequency. It is to be noted that the only thing required of the voltmeter is sensibility, as, in each case, it is set at the same point and is working on the same frequency and wave form. The same is true of the ammeter with 
respect to scale errors and the effect of frequency on its readings, but the wave form of the current changes slightly between readings. Yet, if the ammeter is affected by wave form, the error enters only differentially.

Two values of capacity are required for the determination of two harmonics. It has been found by experiment that the ratios of the two values of capacity employed may conveniently lie between 1.5 and 2, for the cases of nearly sinusoidal and much distorted waves, respectively. With such a ratio, and if $R_{1}$ is kept as low as possible, $R_{2}$ will, roughly, be half of $R_{3}$, thus giving good spacing. As shown below, the use of good paper condensers and soft-iron ammeters will give fairly accurate results on badly distorted waves, but for good waves it is better to use standard mica condensers with a dynamometer-ammeter, and the accuracy may be still further increased if an approximate value of the phase angle of the condensers is known. In case an alternating current mil-ammeter is not available, the series resistance of an alternating current voltmeter, preferably of the dynamometer type, may be short-circuited, giving a mil-ammeter which requires from 0.05 to 0.1 amperes for full scale deflection. The recently introduced type of thermo-ammeter has the advantage of having a negligible inductance and extremely low resistance, but the needle is so slow in assuming its final position that the source must be very steady to get consistent readings. Yet some inductance is of advantage in that it may be used to neutralize the reactance of the condenser, and also to reduce the effect of the residual higher harmonics of which the formulas do not take account. It is necessary in analyzing very good waves that either the inductance of the circuit be made negligible, or that it be made of such a magnitude that its frequency of resonance with the capacity used shall be at least as low as the highest harmonic of which the formulas take account, this being the fifth in equations (5) and (6), above. If this is not done, resonance may occur in the case of some neglected higher harmonic, bringing it out strongly in comparison with the lower ones of which the formula does take account, even though its amplitude in the emf. wave may be negligibly small. It is usually easy and convenient in practice to bring the resonance point between the two harmonics of which account is taken, 
or even, roughly, to approach the conditions of resonance for both $C_{1}$ and $C_{2}$,

$$
\begin{array}{r}
9 p^{2} C_{1} L=\mathrm{I} \\
25 p^{2} C_{2} L=\mathrm{I}
\end{array}
$$

with a single value of $L$. This is the ideal condition for accuracy, although for other practical considerations it is better to take $C_{1}$ and $C_{2}$ rather closer together than this condition requires. In fact, nothing is gained by attempting to make the tuning close.

By making settings on the ammeter and voltmeter well up on the scale, and allowing them to stand some little time with current on, so as to insure steady conditions, the impedance settings may be made to o. I per cent, or slightly better under favorable conditions. Hence, the calculations should be carried to at least as great an accuracy, but this need be done only in the case of $a_{1}, a_{4}$, $a_{7}, m_{3}$, and $m_{6}$, as these are the computed quantities on which the accuracy chiefly depends.

\section{EXPERIMENTAL RESULTS.}

A few other details will be considered in connection with some actual analyses.

In the first case, a badly distorted wave was obtained by connecting in series three machines from a harmonic alternator set, in which the machines are all rigidly connected to the same shaft. The actual ratios of harmonics could be determined separately. The values given are corrected for slight impurities known to be present in the fundamental. Paper condensers and an ammeter of the soft-iron type giving full scale for 0.5 ampere were used in this case. In the following table of the data of the experiment, the harmonics are expressed in per cent of the fundamentals:

$C_{1}=10.00$ microfarads.

$C_{2}=19.32$ microfarads.

$L=$ I0.6 millihenrys. Freq. $=6 \mathrm{r} .2$ cycles per second.
$R_{1}=$ I0.2 ohms.

$R_{2}=85.0$ ohms.

$R_{3}=\mathrm{I} 40.6 \mathrm{ohms}$.

$E=33$ volts.

$I=0.235$ ampere. 


\begin{tabular}{l|c|c}
\hline & $\mathbf{E}_{3}$ & $\mathbf{E}_{\tilde{j}}$ \\
\hline Calculated $\ldots \ldots \ldots \ldots \ldots \ldots \ldots \ldots \ldots \ldots$ & $\begin{array}{c}\text { Percent. } \\
\text { Percent. } \\
17.6\end{array}$ \\
\hline
\end{tabular}

With the same instrument and the same type of condenser, the method was tried with a machine giving a wave approximately sinusoidal. When computed without allowing for the phase angle of the condensers, which averaged I $^{\prime}{ }^{\prime}$ at 60 cycles, $E_{3}$ came out a fairly large imaginary, and $E_{5}$ about 5 per cent. When computed, allowing for the phase angle of the condensers by adding to $R$ the equivalent series resistance, $r=\tan \theta / p C$, due to the dielectric loss, better results were obtained. Here, as in the cases which follow, they are compared with the results of careful analyses, by Dr. M. G. Lloyd, some of which were obtained with a Rosa curve tracer, and others by means of a special rotating commutator designed by Dr. Lloyd and Mr. J. V. S. Fisher. ${ }^{3}$

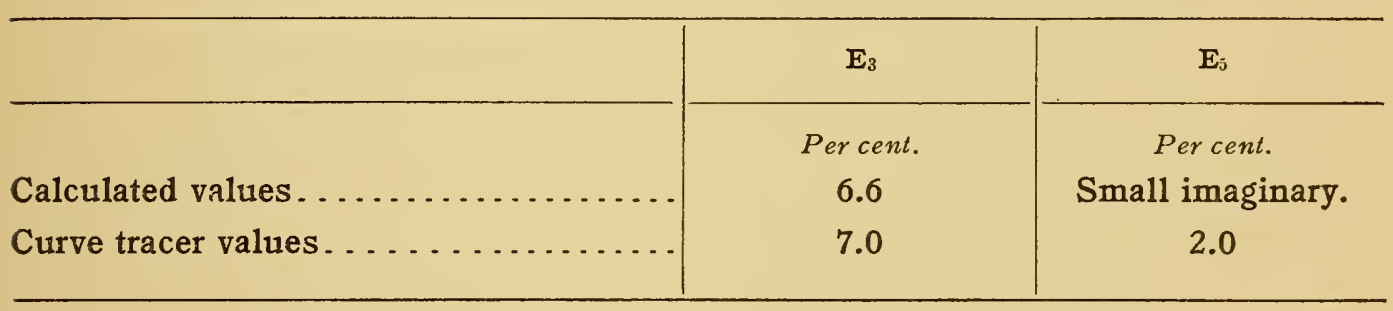

The phase angle of the condensers used was determined for 60 cycles. The same correction for phase was applied to $a_{1}, a_{2}$, and $a_{3}$, similarly the same to each of $a_{4}, a_{5}, a_{6}$, no attempt being made to distinguish between values of $R_{1}$ for the different frequencies. In fact, a careful examination of the equations, or of Fig. 3, will show that the final accuracy depends largely on the determination of $a_{1}, a_{4}$, and $a_{7}$, and consequently on the calculation of $m_{3}$ and $m_{6}$, which depend directly upon them, so that the value of $r$ corresponding to the fundamental may safely be used to correct the higher frequency terms also. 
The following case is that of a composite wave from a 3 -machine motor-generator set, the third being the only harmonic added:

$C_{1}=\mathrm{I} .0037$ microfarads.

$R_{1}=$ I6I ohms.

$L=6 \mathrm{I} .2$ millihenrys.

$R_{2}=2082$ ohms.

$E=198$ volts.

$I=0.08$ ampere.

Frequency $=6 \mathrm{I} .2$ cycles per second.

$E_{3}$ (calculated) Per cent.

$E_{3}$ (actual) 24.6 22.5

The condenser was a mica standard, having a phase angle at 60 cycles of $4^{\prime}$. The calculated value of $E_{3}$ is not changed by allowing for the phase angle of the condenser. The mil-ammeter used was of the dynamometer type, full scale reading o.I ampere, resistance I60 ohms, inductance 61.2 millihenrys.

When used on a machine giving a good emf. wave, 200 millihenrys were added to the circuit containing this mil-ammeter and the mica condensers in order to suppress the residual higher harmonics, as explained above.

\begin{tabular}{|c|c|c|}
\hline & $\mathbf{E}_{3}$ & $\mathbf{E}_{j}$ \\
\hline Calculated values. & $\begin{array}{c}\text { Percent. } \\
7.2\end{array}$ & $\begin{array}{c}\text { Percent. } \\
0\end{array}$ \\
\hline Curve tracer values. & 5.5 & 1 \\
\hline
\end{tabular}

Allowance for dielectric loss was necessary.

A still more rigorous test of the method was made by attempting to analyze the emf. of a machine in which the third harmonic is practically negligible.

\begin{tabular}{|c|c|c|c|}
\hline & $\mathbf{E}_{3}$ & $\mathrm{E}_{j}$ & $\mathbf{E}_{7}$ \\
\hline Values by rotating commutator. & $\begin{array}{c}\text { Percent. } \\
0.6\end{array}$ & $\begin{array}{c}\text { Percent. } \\
1.6\end{array}$ & $\begin{array}{c}\text { Percent. } \\
1.0\end{array}$ \\
\hline $\begin{array}{l}\text { Calculated for third and fifth.. } \\
\text { Calculated for third only..... }\end{array}$ & $\begin{array}{l}\text { Small } \\
\text { imaginary. } \\
4.8\end{array}$ & $\begin{array}{c}\text { Small } \\
\text { imaginary. }\end{array}$ & \\
\hline Calculated for fifth only. . & & 2.1 & \\
\hline
\end{tabular}


Evidently the most the method is capable of in the case of so good a wave is to indicate that the harmonics are extremely small.

But in a large number of engineering problems this is all that is required. As explained above, the small imaginaries indicate that the wave is so nearly sinusoidal that a separation into three components is beyond the accuracy of the method. Either of the above values, rough though they are-namely, 4.8 per cent for the case where all the residuals are lumped together and computed as the third, or 2.I per cent similarly obtained for the fifth-really gives a more adequate description of the wave than to call it exactly sinusoidal. At first sight it seems strange that an equally good or an even better result should be obtained by solving for only one harmonic than by trying to take account of two, but it is to be noticed that in the case of one harmonic only the result depends on but two readings, in one of which the condenser is short circuited while in the other the current is so nearly in quadrature that a small change in the resistance component of the impedance is negligible. Like instances, where an analysis for one harmonic only gives better results than one including two harmonics, may occur in machines of higher frequency.

The following refers to an I8o-cycle machine:

\begin{tabular}{|c|c|c|c|}
\hline & Third & Fifth & Seventh \\
\hline Curve tracer values. & $\begin{array}{c}\text { Percent } . \\
4.0\end{array}$ & $\begin{array}{c}\text { Per cent. } \\
0.4\end{array}$ & $\begin{array}{c}\text { Percent. } \\
0.4\end{array}$ \\
\hline Calculated for third and fifth. & 4.6 & Imaginary. & \\
\hline Calculated for third only.... & 4.3 & & \\
\hline
\end{tabular}

In this, as in the other cases of very good wave form, calculation for one harmonic only has the advantage of being nearly independent of the energy loss in the condenser, the latter making in this particular case a difference of only o. I per cent.

For still higher frequencies, where small values of capacity can be used, it would be advisable to employ an air condenser in order to avoid the uncertainty of correcting for the capacity and phase angle with frequency. In all other cases the condenser should be calibrated at the frequency used. 


\section{CONCLUSION.}

From the foregoing we may say that the accuracy of the method is intermediate between that of the curve tracer and that of the oscillograph, and may be taken as 2 or 3 per cent. of the fundamental.

It has the disadvantage of giving no idea of the phase relations, and hence of the form factor, and of not lending itself to the analysis of current waves, unless a considerable amount of energy is available.

Its chief advantages are that no special apparatus is required and that the calculations are simple. In case it is desired only to know whether or not the wave is nearly pure all the harmonics may be lumped together and computed as if the third were alone present, thus still further simplifying the observations and computations. In special cases a fairly complete knowledge of the impurities may be gained, for example, in the case of a star-connected, 3-phase generator. If the wave be analyzed for the fifth and seventh, the first four odd harmonics are taken account of, since the third and ninth are automatically eliminated by the star connection.

But for the accuracy necessary in commercial work engineers have usually found that the third and the fifth are the only harmonics large enough to be of importance.

Another advantage of the method is that neither direct access to the generator nor the use of a synchronous motor is necessary, so that observations may be taken at a distance from the machine.

I am indebted to Messrs. F. W. Grover and H. L. Curtis for the determination of the inductances, and the capacity and power factor of the condensers used, and to Mr. Isouis Cohen for many valuable suggestions. In fact, the method was suggested by an investigation by Mr. Cohen of the differential damping out of harmonics in an alternating magnetic field, by a copper slab placed in it.

Washington, April 5, igo9. 